\title{
Treatment Outcome of Papillary Carcinoma Confined to the Thyroid Isthmus
}

\author{
Hui Huang, Shao-Yan Liu*, Song Ni, Zong-Min Zhang, Xiao-Lei Wang, Zhen-Gang Xu \\ Department of Head and Neck Surgery, National Cancer Center/Cancer Hospital, Chinese Academy of Medical Sciences, Peking Union \\ Medical College, Beijing, China \\ Email: huanghuinj@163.com, ^shaoyanliu.bj@263.net
}

How to cite this paper: Huang, H., Liu, S.-Y., Ni, S., Zhang, Z.-M., Wang, X.-L. and Xu, Z.G. (2016) Treatment Outcome of Papillary Carcinoma Confined to the Thyroid Isthmus. Journal of Cancer Therapy, 7, 963-969. http://dx.doi.org/10.4236/jct.2016.712093

Received: August 16, 2016

Accepted: November 18, 2016

Published: November 21, 2016

Copyright $\odot 2016$ by authors and Scientific Research Publishing Inc. This work is licensed under the Creative Commons Attribution International License (CC BY 4.0).

http://creativecommons.org/licenses/by/4.0/

\begin{abstract}
Objective: The purpose of this study is to evaluate the clinicopathologic characteristics and treatment outcomes of Papillary Thyroid Carcinomas (PTC) of the isthmus and to establish an appropriate surgical strategy. Methods: Thirty-four patients with PTC in isthmus are managed by surgery in National Cancer Center/Cancer Hospital of Chinese Academy of Medical Sciences, Peking Union Medical College from 19852008. Demographic data, surgical procedures, pathological features, stages and outcomes are analyzed. Results: Seven patients were men and 27 were women. The median age was 41 years (range, 20 - 71). Twenty-five patients were treated with thyroid isthmusectomy or wide field isthmusectomy, five with hemithyroidectomy (lobectomy and isthmusectomy) and four with hemithyroidectomy and partial resection of the contralateral lobe. Twenty-eight patients had a pathologically T1 lesion (pT1); two patients had a pT2 lesion and four had a pT3 lesion. Five patients (14.7\%) had papillary carcinoma detected in one of the pretracheal lymph nodes. Thirty-two patients had a solitary lesion confined to the thyroid isthmus. One patient had two lesions in the thyroid isthmus and another one had two lesions located in the thyroid isthmus and right lobe respectively. With a median follow-up of 94 months (range, 12 - 274), two patients had a recurrence and both survived after a re-operation. There was no regional lymph node or distant organ recurrences. No deaths occurred. Conclusions: Isthmusectomy or wide field isthmusectomy could be a sufficient treatment for PTC confined to the thyroid isthmus. We also recommend that pretracheal lymph node dissection be considered.
\end{abstract}

\section{Keywords}

Thyroid Tumor, Isthmus of Thyroid, Papillary Carcinoma, Surgery

\section{Introduction}

The frequency of papillary carcinoma arising in the thyroid isthmus only has been 
demonstrated within a range of $1 \%$ to $9.2 \%$ [1] [2] [3] [4]. There have been no specific guidelines for the surgical treatment of patients with papillary carcinoma arising in the isthmus [5] [6] [7].

Most patients were treated with completion thyroidectomies after isthmusectomy for nodules [8] [9] [10]. PTCs located in the isthmus are more likely to be associated with multifocal disease, lymph node involvement and capsule invasion than carcinomas in other thyroid regions, and total thyroidectomy is to be considered as an appropriate surgical treatment [1] [4] [11]. However, Nixon et al. suggested that if the nodule was solitary and small in size and confined to the isthmus without evidence of extraglandular extension, the patient may be suitable for thyroid isthmusectomy or wide field isthmusectomy [2]. Sugenoya A. et al. also recommended that isthmusectomy, including an adequate edge of surrounding normal thyroid tissues of each lobe, is sufficient and an appropriate primary surgical procedure for localized differentiated thyroid carcinoma of the isthmus [3]. Thyroid isthmusectomy is defined to remove the entire isthmus and wide field isthmusectomy is to remove the entire isthmus with surrounding thyroid tissues of each lobe. It should be noted that isthmusectomy or wide field isthmussectomy should encompasses the lesion and a surrounding portion of normal tissue. Isthmusectomy has the benefit of avoiding dissection of the recurrent laryngeal nerve and parathyroid glands, thus limiting postoperative complications [2].

There is no consensus on the extent of lymph node dissection that should accompany treatment of isthmic PTC. Studies reported that central lymph node metastasis was present in $40.3 \%$ and $71.1 \%$, and bilateral CND was considered to be needed for isthmic PTC due to the high rate of bilateral central lymph node metastasis especially involving the pretracheal and bilateral paratracheal lymph nodes [1] [12]. Delphian Lymph Node (DLN) metastasis was also reported a high metastasis rate in carcinomas located in the thyroid isthmus [13] [14].

This study is designed to analyze the outcomes of patients with papillary carcinoma in the thyroid isthmus treated with surgery at our institution. The aim is to demonstrate the role of thyroid isthmusectomy in the management of thyroid isthmus cancer.

\section{Methods and Patients}

34 patients with papillary thyroid carcinoma located in isthmus managed primarily by surgery in National Cancer Center/Cancer Hospital, Chinese Academy of Medical Sciences, Peking Union Medical College from 1985-2008 were retrospectively analyzed. All the patients included were suspected with a malignant nodule in the isthmus by preoperative ultrasonography and any patients with suspected malignant nodule(s) in the lateral lobe were excluded. Data were collected from the electronic medical record on patient demographics, types of surgery, and the pathological characteristics. Pathological details included tumor histology, size, number and presence of microscopic extrathyroidal extension. Outcome data included local, regional, or distant recurrence. The presence of recurrence after treatment was based on cytological or histopathological evidence of disease. Distant disease was determined by imaging studies, including 
chest X ray or CT scans. Statistical analysis was performed using SPSS version 20.0 software. Survival outcomes were analyzed using the Kaplan-Meier method.

\section{Results}

The clinical and pathological characteristics are shown in Table 1.

\subsection{Patients and Treatment}

Seven patients were men and 27 were women. The median age was 41 (range, 20 - 71) years. Twenty-five patients had a solitary nodule confined to the thyroid isthmus and nine patients with multiple nodules in thyroid lobes. All patients were with clinically negative lymph node(cN0) except one (2.9\%) with clinically positive lymph node in left level IV (cN1b). All patients were free of distant metastases. Thirty-one patients (91.2\%) were diagnosed with papillary thyroid carcinoma at frozen section pathological analysis and three patients $(8.8 \%)$ were diagnosed at final histopathological analysis. Twenty-five patients were treated with thyroid isthmusectomy or wide field isthmusectomy, five with hemithyroidectomy (lobectomy and isthmusectomy) and four with hemithyroidectomy and partial resection of the contralateral lobe. Ten patients received pretracheal lymph node dissection ( 2 with DLN resected). The patient with cN1b received unilateral modified neck dissection and was confirmed with lymph node metastasis at final histopathological analysis ( $\mathrm{pN} 1 \mathrm{~b})$. No patients received postoperative

Table 1. Clinical and pathological characteristics.

\begin{tabular}{cc}
\hline Variables & Number (\%) \\
\hline Age (year) & $19(55.9)$ \\
$<45$ & $15(44.1)$ \\
Sex & \\
Male & $7(20.6)$ \\
Female & $27(79.4)$ \\
pT stage & \\
T1 & $28(82.4)$ \\
T2 & $2(5.9)$ \\
T3 & $4(11.8)$ \\
pN stage & \\
N0 & $29(85.3)$ \\
N1a & $4(11.8)$ \\
N1b & $1(2.9)$ \\
Capsular invasion & \\
No & $18(52.9)$ \\
Yes & $167.1)^{*}$ \\
\hline &
\end{tabular}

${ }^{\star}$ Four of the 16 patients with extrathyroidal extension (ETE). 
radioiodine treatment.

\subsection{Pathology}

All of the malignant nodules were diagnosed papillary carcinoma. Twelve patients accompanied with nodular goiter and two with chronic lymphocytic thyroiditis. Four patients were diagnosed with extrathyroidal extension after paraffin histopathologic analysis, and thirteen patients with microscopic capsular invasion. Thirty-two patients had a solitary lesion confined to the thyroid isthmus. One patient had two lesions in the thyroid isthmus and one had two lesions located in the thyroid isthmus and right lobe respectively. The median size of the lesion was 1 (range, $0.3-4) \mathrm{cm}$. Twenty-eight patients had a pathologically T1 disease (pT1), two patients had a pT2 lesion and four had a pT3 lesion. Five patients (14.7\%) had papillary carcinoma detected in one of the pretracheal lymph nodes and one of them accompanied with metastatic lymph node in left level IV (1/7). All of the five patients with pretracheal lymph node metastasis had a solitary isthmic lesion and only one had a pT3 lesion.

\subsection{Outcomes}

There were no complications of recurrent laryngeal nerve palsy or hypocalcemia. One patient was lost-to-follow up. The median follow-up time of the other thirty-three patients was 94 months (range, 12 - 274). During the follow-up time, two patients had a recurrence and both survived after a re-operation. One who originally had two isthmic lesions (pT1N0) recurred at fifty-seven months and the histopathologic examination confirmed carcinosarcoma in left lobe after total thyroidectomy. Another patient (pT1N0) recurred at 101 months and the histopathologic examination confirmed papillary carcinoma after left lobectomy. Both patients survived to the follow-up deadline (67 and 274, respectively). There was no regional lymph node or distant organ recurrences. The 10-year recurrence-free survival rate was $89.4 \%$ (Figure 1 ). No deaths occurred. The 10 -year overall survival rate was $100 \%$.

\section{Discussions}

The frequency of papillary carcinoma arising in the thyroid isthmus is low (1\% to 9.2\%) [1] [2] [3] [4], and there are considerable literature debates on the extent of the surgical resection. No consensus existed on the appropriate management of thyroid cancer confined to the isthmus [5] [6] [7]. Papillary carcinoma arising in the isthmus has been reported more likely to be associated with multifocal lesions, lymph node metastasis and capsular invasion, and total thyroidectomy was considered as an appropriate surgical treatment [1] [4] [11]. A completion total thyroidectomy is normally performed in most studies [8] [9] [10]. Only a few studies advocated isthmusectomy or wide field isthmusectomy as a suitable and reasonable surgical procedure for isthmic PTC. In Sugenoya et al's report on 19 well-differentiated thyroid isthmus malignancies, 4 patients were treated with isthmusectomy alone and all were alive with no evidence of recurrence 20 years after surgery [3]. Nixon et al. reported that if the nodule was solitary and 


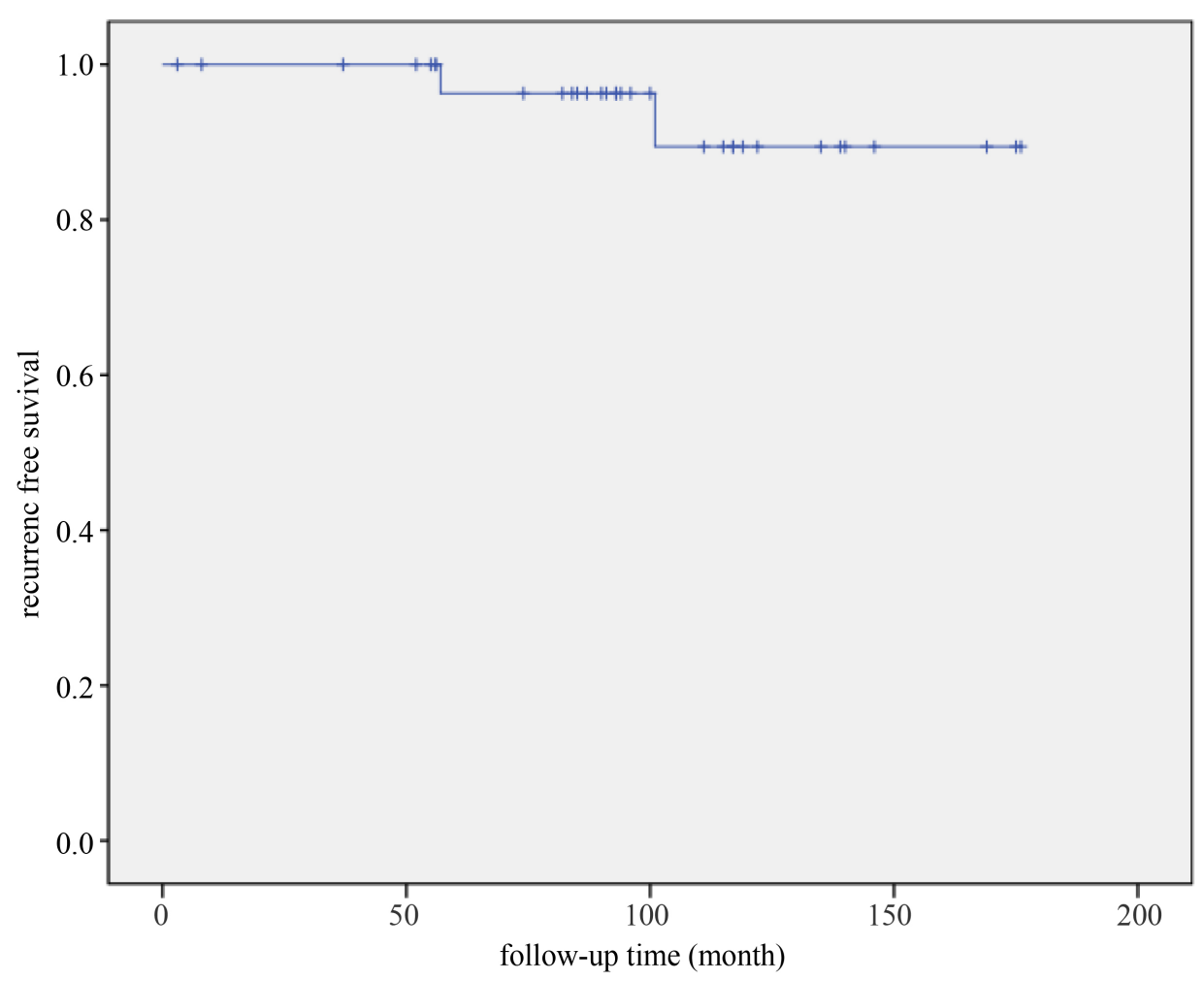

Figure 1. The 10-year recurrence-free survival of the patients.

small in size and confined to the isthmus without evidence of extrathyroidal extension, the patient may be suitable for thyroid isthmusectomy or wide field isthmusectomy with an excellent survival (10-year recurrence-free survival was 100\%) [2]. The American Thyroid Association did not report isthmusectomy as an appropriate surgical procedure for papillary thyroid carcinoma [5]. In our study, only two of the patients treated with isthmusectomy had a recurrence and both survived after a re-operation. Most patients did not recur even with lymph node metastasis or extrathyroidal extension. Our results suggested that isthmusectomy or wide field isthmusectomy can be an effective treatment for isthmic PTC.

There is no consensus on the extent of lymph node dissection that should accompany with the treatment of isthmic PTC. The lymphatic system of the thyroid gland is parallel to the venous drainage system [15], and drains inferiorly into the pretracheal and paratracheal lymph nodes, and follows the inferior jugular lymphatics and superior mediastinal lymphatics [16], while the isthmus and upper lobes of the thyroid are drained superiorly to the prelaryngeal lymph nodes [13] [17]. In Song CM, et al.'s study, the most common subsite of central compartment metastasis in isthmic PTC was the pretracheal lymph node, and the isthmic PTCs had higher rates of metastasis to pretracheal and bilateral paratracheal lymph nodes than the non-isthmic PTCs, and complete bilateral central neck dissection should be considered [12]. In our study, only ten patients had pretracheal lymph node dissection done and no patients had bilateral paratracheal lymph node dissected. Though five patients had pretracheal lymph node 
metastasis, no patient had a lymph node recurrence during the follow-up time. We suggest that pretracheal lymph node dissection is needed and also sufficient for most isthmic PTCs with clinically negative lymph node. The DLN is located in the fascia above the isthmus of the thyroid gland, between the cricoid and thyroid cartilage; it is also known as the prelaryngeal, precricoid, or cricothyroid node [18] [19]; is known to be predictive of a poor prognosis [20]. DLN metastasis was associated with tumor location in the isthmus or upper third of the thyroid, and with ETE [13]. DLN should be resected with pretracheal lymph node, especially for those with lymph node metastasis or with tumor ETE.

Our results suggest that isthmusectomy/wide field isthmusectomy could be sufficient treatment for PTC confined to the thyroid isthmus. We also recommend that the pretracheal lymph node dissection routinely be performed.

\section{References}

[1] Lee, Y.S., Jeong, J.J., Nam, K.H., Chung, W.Y., Chang, H.S. and Park, C.S. (2010) Papillary Carcinoma Located in the Thyroid Isthmus. World Journal of Surgery, 34, 36-39. https:/doi.org/10.1007/s00268-009-0298-6

[2] Nixon, I.J., Palmer, F.L., Whitcher, M.M., et al. (2011) Thyroid Isthmusectomy for WellDifferentiated Thyroid Cancer. Annals of Surgical Oncology, 18, 767-770. https:/doi.org/10.1245/s10434-010-1358-8

[3] Sugenoya, A., Shingu, K., Kobayashi, S., et al. (1993) Surgical Strategies for Differentiated Carcinoma of the Thyroid Isthmus. Head \& Neck, 15, 158-160.

https:/doi.org/10.1002/hed.2880150212

[4] Karatzas, T., Charitoudis, G., Vasileiadis, D., et al. (2015) Surgical Treatment for Dominant Malignant Nodules of the Isthmus of the Thyroid Gland: A Case Control Study. International Journal of Surgery, 18, 64-68. https:/doi.org/10.1016/j.ijsu.2015.04.039

[5] Haugen, B.R., Alexander, E.K., Bible, K.C., et al. (2015) American Thyroid Association Management Guidelines for Adult Patients with Thyroid Nodules and Differentiated Thyroid Cancer: The American Thyroid Association Guidelines Task Force on Thyroid Nodules and Differentiated Thyroid Cancer. Thyroid, 26, 1-133. https:/doi.org/10.1089/thy.2015.0020

[6] Watkinson, J.C. (2004) The British Thyroid Association Guidelines for the Management of the Thyroid Cancer in Adults. Nuclear Medicine Communications, 25, 897-900. https:/doi.org/10.1097/00006231-200409000-00006

[7] Pacini, F., Schlumberger, M., Dralle, H., et al. (2006) European Thryoid Cancer Taskforce. European Consensus for the Management of Patients with Differentiated Thyroid Carcinoma of the Follicular Epithelium. European Journal of Endocrinology, 154, 787-803. https:/doi.org/10.1530/eje.1.02158

[8] Maser, C., Donovan, P. and Udelsman, R. (2007) Thyroid Isthmusectomy: A Rarely Used But Simple, Safe, and Efficacious Operation. Journal of the American College of Surgeons, 204, 512-514. https:/doi.org/10.1016/j.jamcollsurg.2006.12.018

[9] Perez-Ruiz, L., Ros-Lopez, S., Gudelis, M., et al. (2008) Isthmectomy: A Conservative Operation for Solitary Nodule of the Thyroid Isthmus. Acta Chirurgica Belgica, 108, 699-701. https:/doi.org/10.1080/00015458.2008.11680319

[10] Skilbeck, C., Leslie, A. and Simo, R. (2007) Thyroid Isthmusectomy: A Critical Appraisal. The Journal of Laryngology \& Otology, 121, 986-989. 
https:/doi.org/10.1017/S0022215106005238

[11] Goldfarb, M., Rodgers, S.S. and Lew, J.I. (2012) Appropriate Surgical Procedure for Dominant Thyroid Nodules of the Isthmus $1 \mathrm{~cm}$ or Larger. Archives of Surgery, 147, 881-884. https:/doi.org/10.1001/archsurg.2012.728

[12] Song, C.M., Lee, D.W., Ji, Y.B., et al. (2015) Frequency and Pattern of Central Lymph Node Metastasis in Papillary Carcinoma of the Thyroid Isthmus. Head \& Neck, 38, E412-E416. https:/doi.org/10.1002/hed.24009

[13] Chai, Y.J., Kim, S.J., Choi, J.Y., et al. (2014) Papillary Thyroid Carcinoma Located in the Isthmus or Upper Third Is Associated with Delphian Lymph Node Metastasis. World Journal of Surgery, 38, 1306-1311. https:/doi.org/10.1007/s00268-013-2406-X

[14] Lee, Y.C., Shin, S.Y., Kwon, K.H., et al. (2013) Incidence and Clinical Characteristics of Prelaryngeal Lymph Node Metastasis in Papillary Thyroid Cancer. European Archives of Oto-Rhino-Laryngology, 270, 2547-2550. https:/doi.org/10.1007/s00405-013-2471-7

[15] Mohebati, A. and Shaha, A.R. (2012) Anatomy of Thyroid and Parathyroid Glands and Neurovascular relations. Clinical Anatomy, 25, 19-31. https:/doi.org/10.1002/ca.21220

[16] Panje, W.R. and Herberhold, C. (1995) The Lymphatic System of the Thyroid and Its Management. Head and Neck Surgery, 3, 443-444.

[17] Nakayama, M., Seino, Y., Okamoto, M., Mikami, T., Okamoto, T. and Miyamoto, S. (2011) Clinical Significance of Positive Delphian Node in Supracricoid Laryngectomy with Cricohyoidoepiglottopexy. Japanese Journal of Clinical Oncology, 41, 987-991. https:/doi.org/10.1093/jjco/hyr091

[18] Iyer, N.G., Shaha, A.R., Ferlito, A., et al. (2010) Delphian Node Metastasis in Head and Neck Cancers-Oracle or Myth? Journal of Surgical Oncology, 102, 354-358. https:/doi.org/10.1002/jso.21640

[19] Thaler, E.R., Montone, K., Tucker, J., et al. (1997) Delphian Lymph Node in Laryngeal Carcinoma: A Whole Organ Study. Laryngoscope, 107, 332-334. https:/doi.org/10.1097/00005537-199703000-00010

[20] Iyer, N.G., Kumar, A., Nixon, I.J., et al. (2011) Incidence and Significance of Delphian Node Metastasis in Papillary Thyroid Cancer. Annals of Surgery, 253, 988-991. https:/doi.org/10.1097/SLA.0b013e31821219ca for you:

Accepting pre-submission inquiries through Email, Facebook, LinkedIn, Twitter, etc. A wide selection of journals (inclusive of 9 subjects, more than 200 journals)

Providing 24-hour high-quality service

User-friendly online submission system

Fair and swift peer-review system

Efficient typesetting and proofreading procedure

Display of the result of downloads and visits, as well as the number of cited articles

Maximum dissemination of your research work

Submit your manuscript at: http://papersubmission.scirp.org/

Or contact jct@scirp.org 